\title{
Desenvolvimento de um Modelo Matemático com Atrito Não Linear para o Pêndulo Simples
}

\author{
Mauri J. Klein, Leonardo B. Maraschin \\ Departamento de Ciências Exatas e Engenharias (DCEEng), UNIJUí \\ 98700-000, Ijuí, RS \\ E-mail: mauri.klein@unijui.edu.br; leonardo.maraschin@unijui.edu.br \\ Antonio C. Valdiero, Luiz A. Rasia \\ Departamento de Ciências Exatas e Engenharias (DCEEng), UNIJUÍ Campus Panambi \\ 98280-000, Panambi, RS \\ E-mail: valdiero@unijui.edu.br; rasia@unijui.edu.br
}

\begin{abstract}
Resumo: Este artigo apresenta um novo modelo matemático de um sistema mecânico do tipo pêndulo simples com validação experimental do mesmo. O objetivo é descrever a seqüência de passos utilizados na formulação matemática do modelo, comparando dados experimentais com modelos da literatura e a partir destes, desenvolver um modelo pertinente com a realidade. Como resultados, têmse a identificação experimental, as modificações do modelo e sua validação nas condições de um pêndulo simples considerando todas as forças atuantes sobre o mesmo.
\end{abstract}

Abstract: This paper presents a new mathematical model for the simple pendulum mechanical system and its experimental validation. The aim is to describe the sequence of steps used in the mathematical formulation of the model by comparing experimental data with literature and from these, develop a relevant and realistic model. The contributions of this work are the identification experimental modifications of the model and its validation in terms of a simple pendulum considering all the forces acting on it.

\section{INTRODUÇÃO}

Os sistemas físicos apresentam-se como não lineares e o seu comportamento pode ser descrito por uma equação diferencial. No entanto, se o funcionamento de um sistema não linear é numa faixa de domínio consideravelmente pequena e próxima a um ponto de equilíbrio, então o mesmo pode se aproximar do comportamento de um sistema linear, cuja dinâmica é descrita por um conjunto de equações diferenciais lineares [4]. No caso do pêndulo simples, a linearidade pode ser constatada quando o sistema está próximo ao seu ponto de equilíbrio.

Neste trabalho será mostrado que o modelo matemático linear e o não linear do pêndulo simples, não representam corretamente o seu movimento oscilatório quando o ângulo inicial for maior que 10 graus, através da comparação dos gráficos gerados com base neles e com o do experimento.

Como o pêndulo é um sistema mecânico presente tanto no estudo de novas tecnologias como em pesquisas científicas, sendo o mesmo utilizado para descrever diferentes fenômenos relacionados com oscilações, bifurcações e caos [2] e também é usado na medição do tempo, em dispositivos de estabilização e na balística [3], será deduzida a equação diferencial que melhor descreva o deslocamento dele, usando as leis da física. Pretende-se contribuir para a solução deste problema com a modelagem matemática e a análise dos resultados de simulação computacional.

\section{METODOLOGIAS}

Para a validação do modelo, iniciou-se com a obtenção dos dados através da bancada experimental do pêndulo simples instalada no laboratório de engenharia mecânica no campus da UNIJUÍ em Panambi-RS, que é composta por uma haste ao qual está preso o pêndulo com comprimento regulável; um sensor que capta a oscilação do pêndulo; uma placa eletrônica dSPACE e um microcomputador ligado à bancada, onde são gravados os dados do sensor. 
Os dados gerados pelas simulações na bancada, conforme Figura 1, foram tratados (processados) para deixá-los em forma de vetores, tornando-os manipuláveis pelo software MATLAB, onde foram armazenados em forma de estrutura.

Os vetores para geração dos gráficos relativos a cada experimento são provindos desta estrutura. Com isto temos a possibilidade de fazer as simulações computacionais em qualquer outro microcomputador.
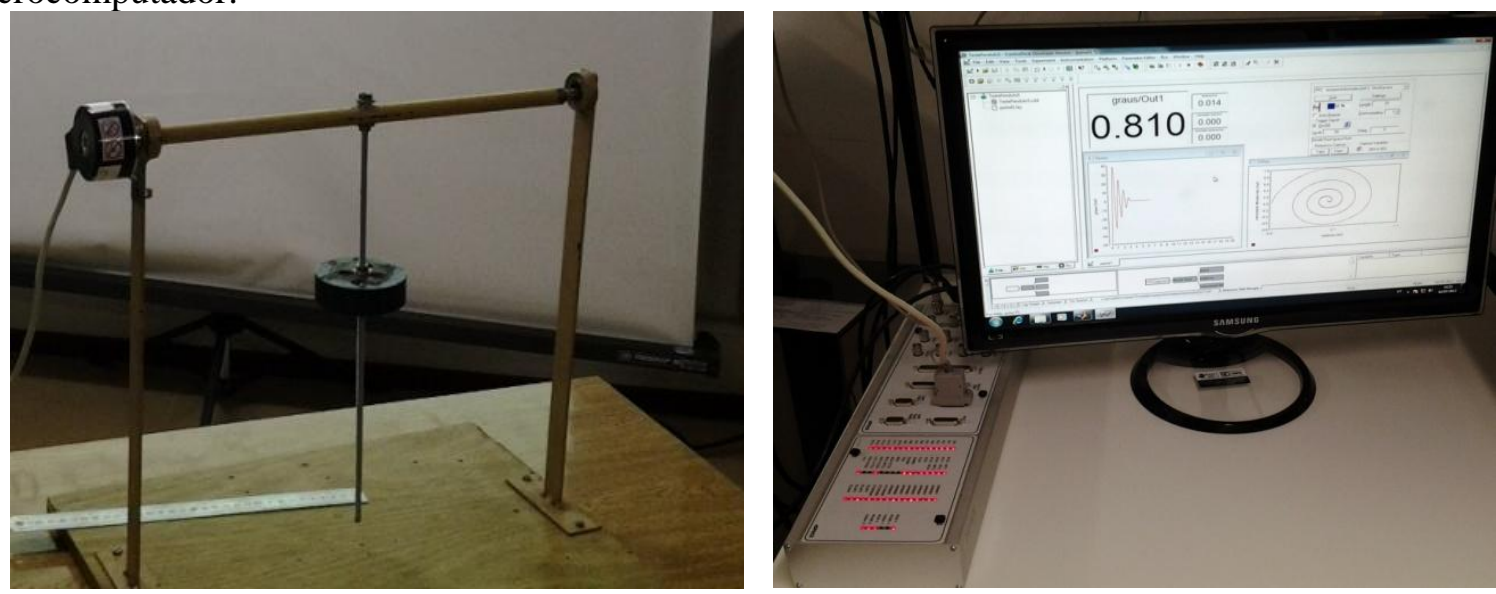

Figura 1: Foto da bancada do pêndulo simples utilizada para validação do modelo.

Para a construção dos diagramas de blocos e simulação computacional foi utilizado o software MATLAB/SIMULINK, com aplicação do método numérico Runge-Kutta de ordem 4 com um passo de integração de 1e-5 e o período analisado em cada simulação de 10 segundos.

\section{DESENVOLVIMENTO DO MODELO MATEMÁTICO}

A modelagem de um pêndulo simples tem como objetivo definir os parâmetros e as variáveis que estão no problema, com posterior dedução da equação diferencial de segunda ordem correspondente, que demonstre a trajetória oscilatória real de um pêndulo.

O pêndulo simples é um sistema físico constituído de um corpo de massa m suspensa por um fio ou por outros elementos de comprimento L. A sua oscilação se dá devido ao efeito da gravidade quando o mesmo for afastado da sua posição de equilíbrio, conforme Figura 2 [5].

Para simplificar o modelo matemático consideramos que a massa do fio não altera o movimento oscilatório do pêndulo e que o fio não é flexível, mantendo com isto, o comprimento desejado.

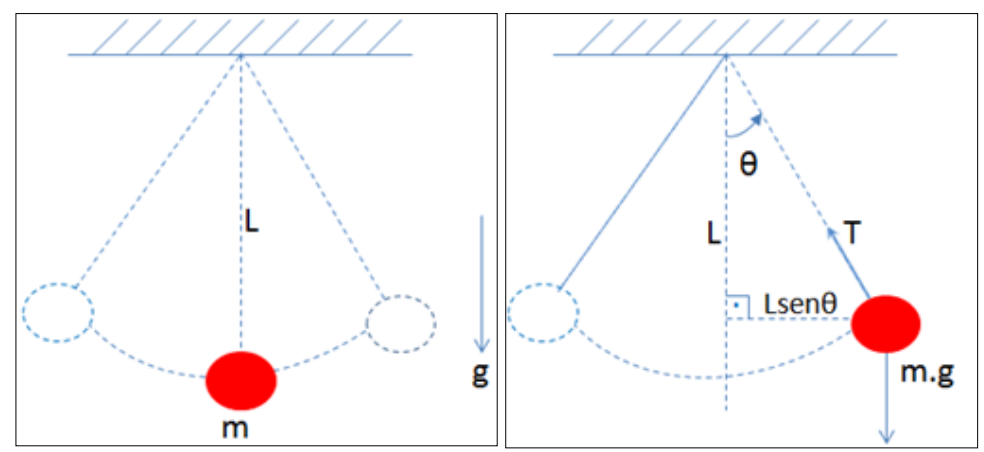

Figura 2 - Desenho esquemático do pêndulo simples com seus parâmetros e variáveis.

Os parâmetros e variáveis do sistema modelado foram obtidos através de medições feitas no pêndulo do experimento e por aceitação de definições da literatura: $\mathrm{g}=9,81 \mathrm{~m} / \mathrm{s}^{2}$ (força 
gravitacional), $\mathrm{L}=0.185 \mathrm{~m}$ (comprimento do pêndulo em metros), $\mathrm{m}=0.825 \mathrm{~kg}$ (massa do pendulo em quilogramas), dTetai $=0 \mathrm{rad} / \mathrm{s}$ (velocidade angular inicial), Tetai $=60 / 180 * \mathrm{PI} \mathrm{rad}$ (posição angular inicial) e o parâmetro B (coeficiente de amortecimento viscoso) é calculado a partir dos dados anteriores.

Para o modelo matemático não linear descrito neste artigo, foi necessária a definição de mais alguns parâmetros: $\sigma_{0}=10^{5} \mathrm{~N} \cdot \mathrm{m}^{-1}$ (coeficiente de rigidez das deformações microscópicas); $\sigma_{2}=\mathrm{B}$ (coeficiente de amortecimento viscoso); alfa $=1$ (representação do atrito estático); $\mathrm{F}_{\mathrm{s}}=$ $0.052 \mathrm{~N}$ (força de atrito estático); $\mathrm{dYs}=0.01 \mathrm{rad} / \mathrm{s}$ (velocidade de Striberk). Os parâmetros $T_{a t r}(\dot{\theta})$ (torque de atrito), $\sigma_{1}$ (coeficiente de amortecimento ), $\mathrm{F}_{\mathrm{c}}$ (força de atrito de Coulomb) e $g_{s s}(\dot{\theta})$ (função de atrito em regime permanente) são calculados. Foi definida também a variável $\mathrm{Z}$ (deformação média que ocorre entre as superfícies) para este modelo, sendo que a sua condição inicial é 0 .

Para $\sigma_{0}, F_{s}$, alfa,dYs foram atribuídos valores buscando fazer com que este modelo matemático demonstrasse o mais próximo possível a oscilação real do pêndulo simples.

\subsection{Formulação matemática}

Quando um pêndulo é deslocado do seu ponto de equilíbrio, forças externas passam a agir sobre o mesmo, entre elas está um torque restaurador devido ao peso do corpo buscando restaurar o pêndulo para sua posição de equilíbrio, com isso temos que:

$$
\sum T=I v
$$

onde:

$$
\begin{gathered}
I=\int r^{2} d m=L^{2} m \\
v=\ddot{\theta} \\
\sum T=-m g L \operatorname{sen} \theta-B \dot{\theta}
\end{gathered}
$$

$\log 0$,

$$
\begin{gathered}
-m g L \operatorname{sen} \theta-B \dot{\theta}=L^{2} m \ddot{\theta} \\
L^{2} m \ddot{\theta}+m g L \operatorname{sen} \theta+B \dot{\theta}=0
\end{gathered}
$$

dividindo por $\mathrm{mL}^{2}$, obtemos:

$$
\ddot{\theta}+\frac{B}{m L^{2}} \dot{\theta}+\frac{g}{L} \operatorname{sen} \theta=0
$$

A EDO (7) é o modelo matemático não linear do pêndulo simples. Se o ângulo $\theta \leq 10^{\circ}$, podemos considerar que $\operatorname{sen}(\theta)=0$, pois o erro será pequeno e assim obtemos a seguinte equação:

$$
\ddot{\theta}+\frac{B}{m L^{2}} \dot{\theta}+\frac{g}{L} \theta=0
$$

A EDO (8) é o modelo matemático linear do pêndulo. Para calcular o valor do B, podemos comparar a equação (8) com a equação movimento com um grau de liberdade $[1,2]$, que está logo abaixo:

$$
\ddot{Y}+2 W_{n} \xi \dot{Y}+W_{n}^{2} Y=0
$$

Sendo que $W_{n}$ é a frequência angular natural e $\xi$ o fator de amortecimento, observamos que:

$$
\begin{aligned}
& W_{n}^{2}=\frac{g}{L} \\
& W_{n}=\sqrt{\frac{g}{L}}
\end{aligned}
$$




$$
\begin{aligned}
& 2 W_{n} \xi=\frac{B}{m L^{2}} \\
& B=2 m L^{2} W_{n} \xi
\end{aligned}
$$

Sendo $\xi$ obtido através da próxima equação:

$$
\xi=\frac{1}{\sqrt{1+(2 \pi / \gamma)^{2}}}
$$

se $\xi<0.1$, então

$$
\xi=\frac{\gamma}{2 \pi}
$$

Sendo que $\gamma$ é calculado usando o logaritmo natural da razão entre duas amplitudes sucessivas do período do pêndulo mostrado na equação abaixo:

$$
\gamma=\ln \left(\frac{x_{1}}{x_{2}}\right)
$$

No modelo matemático não linear desenvolvido do pêndulo simples foi substituído $B \dot{\theta}$ pelo $T_{a t r}(\dot{\theta})$ [5], conforme está abaixo:

sendo que,

$$
\ddot{\theta}+\frac{T_{a t r}(\dot{\theta})}{m L^{2}}+\frac{g}{L} \operatorname{sen} \theta=0
$$

onde:

$$
T_{a t r}(\dot{\theta})=\sigma_{0} Z+\sigma_{1} \dot{Z}+\sigma_{2} \dot{\theta}
$$

$$
\begin{gathered}
\sigma_{1} \leq \frac{\sigma_{2}}{\left(\frac{F_{s}}{F_{c}}-1\right)} \\
\sigma_{2}=B \\
\dot{Z}=\dot{\theta}\left(1-\frac{\text { alfa } * \sigma_{0} \operatorname{Sign}(\dot{\theta}) Z}{g_{s s}(\dot{\theta})}\right)
\end{gathered}
$$

e,

$$
\begin{gathered}
g_{s s}(\dot{\theta})=F_{c}+\left(F_{s}-F_{c}\right) e^{-\left(\frac{\dot{\theta}}{d Y s}\right)^{2}} \\
F_{c}=0,5 * F_{s}
\end{gathered}
$$

\section{RESULTADOS}

Para a simulação computacional dos modelos propostos, foram utilizados alguns diagramas de blocos (Figuras 3 e 4), com distintos parâmetros a fim de ilustrar a discrepância entre um modelo e outro, e também em relação ao modelo experimental.
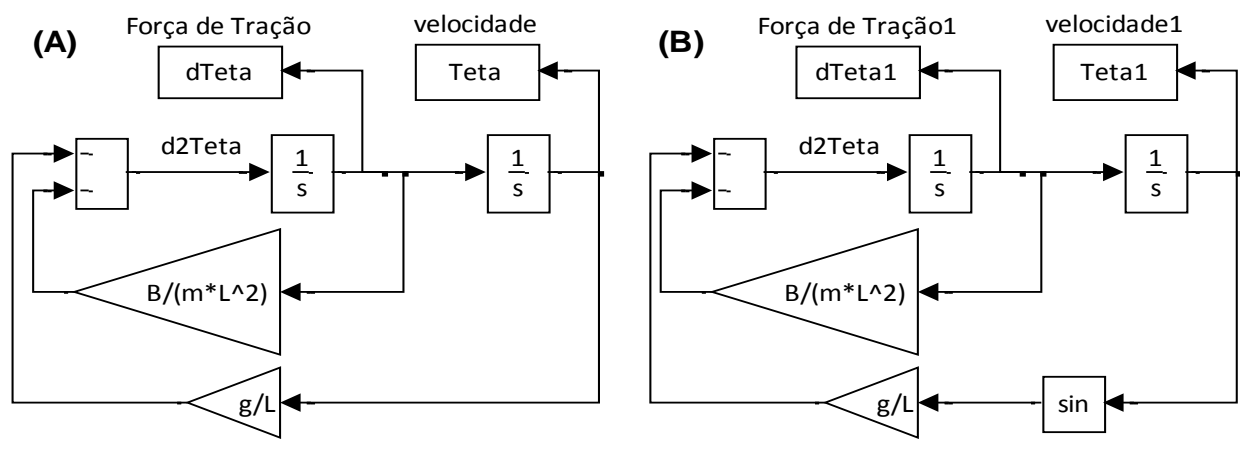
Figura 3: Diagrama de blocos do modelo matemático linear(A) e não linear(B) do pêndulo simples.
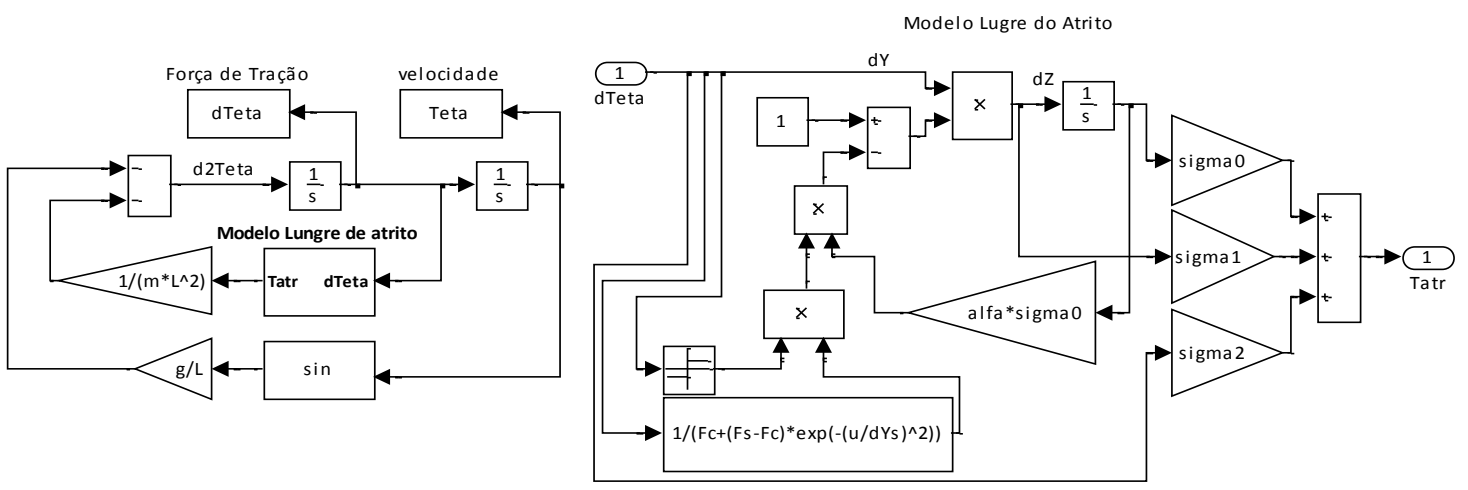

Figura 4: Diagrama de blocos do modelo matemático não linear desenvolvido do pêndulo utilizando modelo Lugre de Atrito.

\subsection{Resultados de validação experimental}

A posição angular inicial de 60 graus e a velocidade inicial de $0 \mathrm{rad} / \mathrm{s}$, foram utilizadas na construção dos gráficos do experimento (Figura 5), na comparação dos modelos linear, não linear e também na validação do modelo não linear desenvolvido.
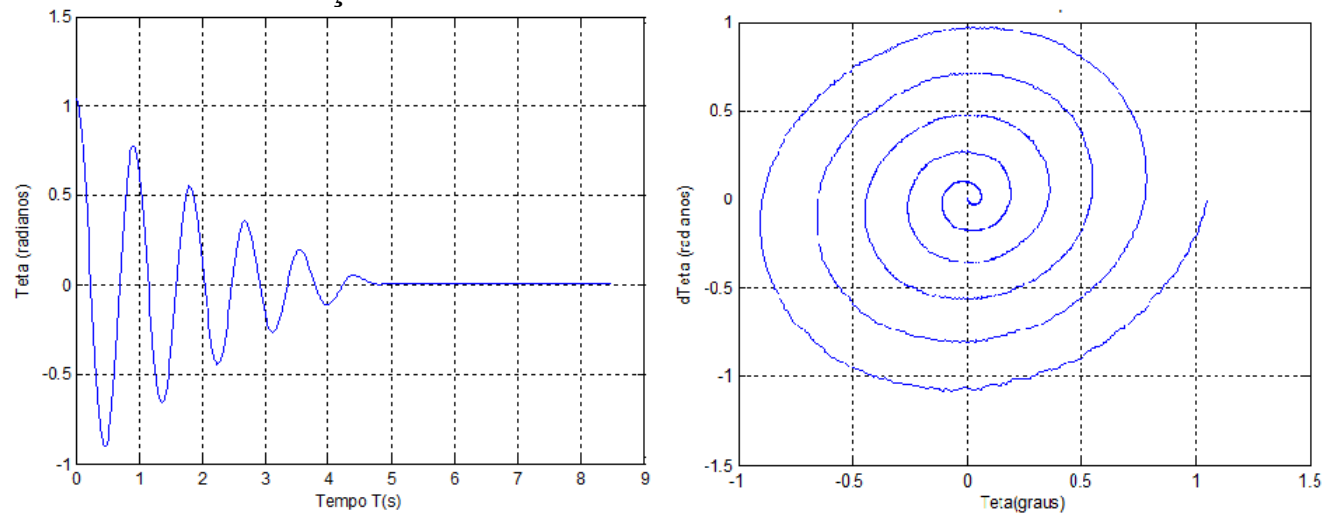

Figura 5: Oscilação do pêndulo em relação ao tempo e plano de fase, gerados a partir dos dados obtidos do experimento.

Apresentados os gráficos obtidos do experimento feito, podemos compará-los com os gráficos da simulação computacional (Figura 6), partindo dos dados da seção 3 e utilizando os diagramas de blocos do Simulink mostrados no início desta seção.
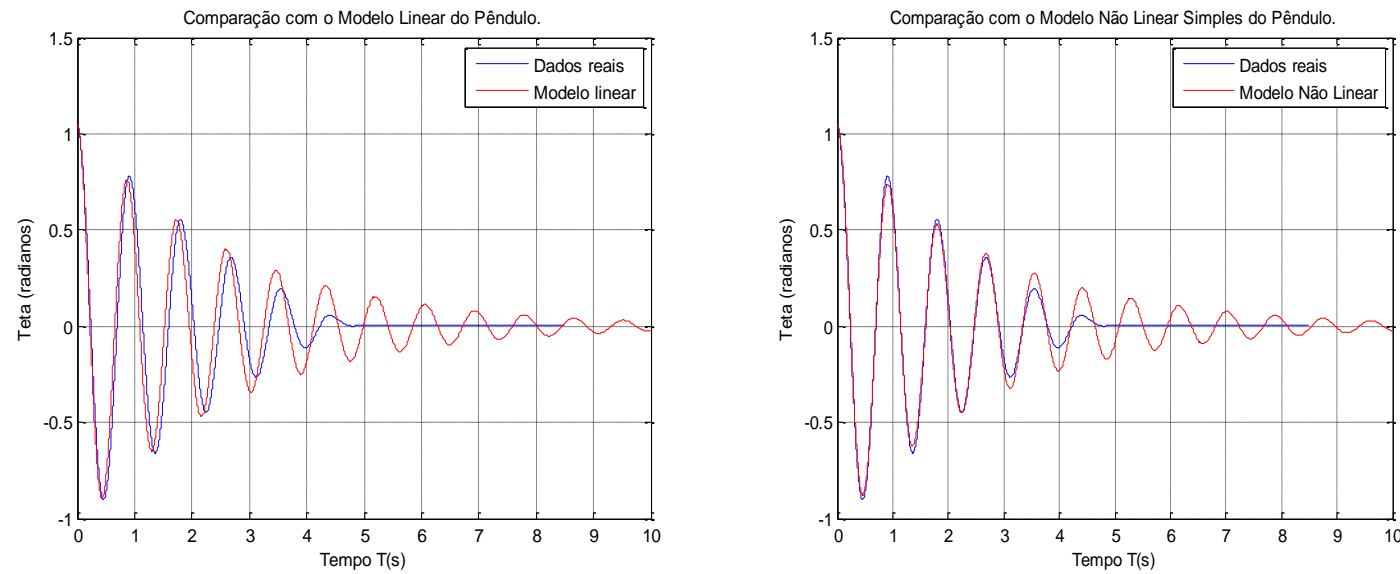

Figura 6: Comparação dos modelos linear e não linear do pêndulo com dados reais 
Percebe-se uma grande diferença entre os dados reais se comparados aos modelos apresentados na literatura. A seguir está a comparação com o modelo matemático desenvolvido, utilizando os parâmetros e variáveis obtidos através de medições e alguns deles calculados.

No primeiro gráfico da Figura 7 percebe-se ainda uma diferença considerável na comparação dos dois gráficos. Passou-se então para uma aproximação através de simulações computacionais, a partir do ajuste em dois parâmetros $\left(B\right.$ e $F_{c}$ ) do modelo matemático desenvolvido, achando assim a melhor aproximação para os mesmos: $B=0.0083$ N.s.m ${ }^{-1} \mathrm{e}$ $\mathrm{F}_{\mathrm{c}}=0.899 * \mathrm{~F}_{\mathrm{s}}$.
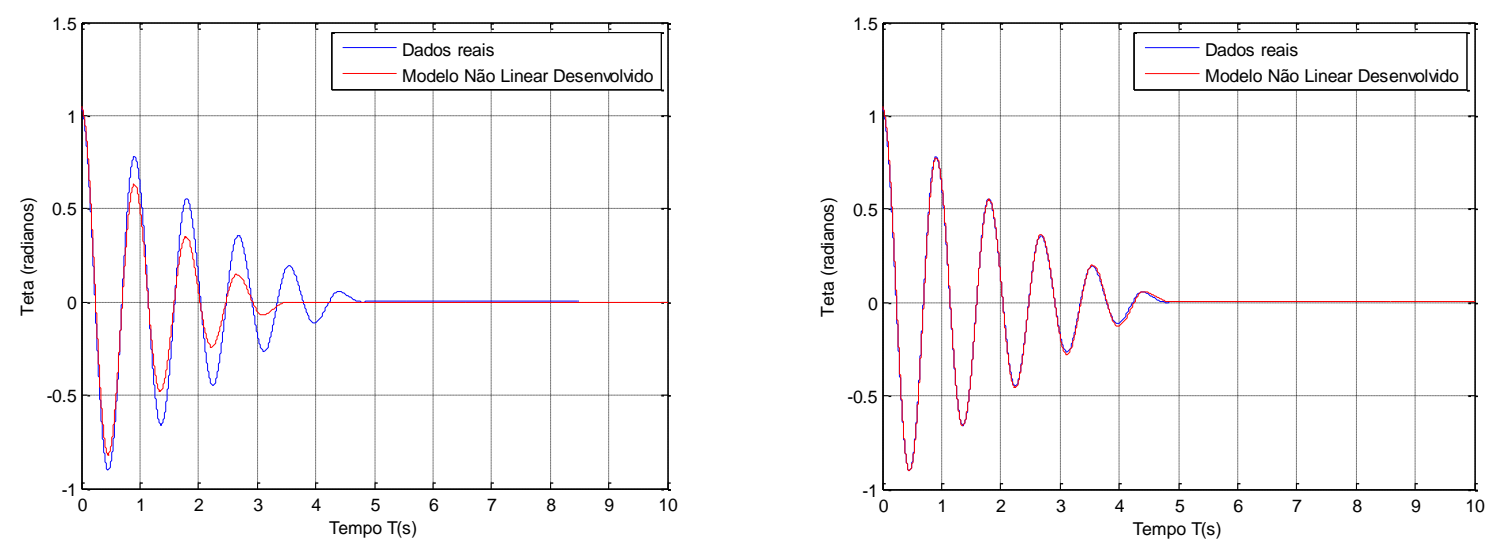

Figura 7: Comparação do modelo matemático não linear desenvolvido com os dados reais,

(a)sem ajuste e (b) com ajuste de parâmetros através de simulações computacionais.

Com isto obteve-se o segundo gráfico da Figura 7 praticamente idêntico ao real, validando desta forma o modelo matemático não linear desenvolvido para o pêndulo simples.

\section{CONCLUSÕES}

Analisando os três modelos matemáticos do pêndulo simples, observa-se que somente o modelo não linear desenvolvido apresenta um resultado próximo do real. Contudo foram necessários alguns ajustes em dois parâmetros de entrada. Para trabalhos futuros, sugere-se a formulação de uma relação entre o comprimento do pêndulo e o valor destas duas variáveis.

\section{REFERÊNCIAS}

[1] L. A. da Fonseca Júnior, J. S. Borges, R. A. Borges, Modelagem e projeto ótimo de sistemas mecânicos lineares e não lineares, em "Simpósio de matemática e matemática industrial", Departamento de Matemática - CAC/UFG, Catalão, 2011.

[2] A. S. de Paula, "Controle de caos multiparâmetros a partir do método ogy: aplicação em um pêndulo não-linear", Dissertação de Mestrado, COPPE - Ufrj, 2007.

[3] M. A. Savi, "Dinâmica não-linear e caos”, E-papers, Rio de Janeiro, 2006.

[4] J.-J. E. Slotine, W. Li, “Applied nonlinear control”, Prentice-Hall, Englewood Cliffs, New Jersey, 1991.

[5] A. C. Valdiero, “Modelagem matemática de robôs hidráulicos”, Editora Unijuí, Ijuí, 2012. 